\title{
Miopatía Inflamatoria: Recuperación funcional desde la medicina biorreguladora. Reporte de caso.
}

\author{
Heidy Marcela Barreto Castañeda, ${ }^{1}$ Yohana Garrido Caballero ${ }^{2}$ \\ Corresponding Author: Heidy Marcela Barreto Castañeda, infodramarcelabarreto@gmail.com \\ 1 infodramarcelabarreto@gmail.com \\ 2 garrido.yohana1920@gmail.com
}

\begin{abstract}
Las miopatías inflamatorias son trastornos heterogéneos que comparten la injuria muscular inmunomediada como característica común; cuenta con un amplio espectro clínico que pueden ir desde manifestaciones a nivel de la piel, tracto gastrointestinal, articulaciones y pulmones. A continuación, se expone el caso de una paciente tratada con medicina biorreguladora por cuadro de miopatía inflamatoria encontrando un gran impacto en su recuperación funcional.
\end{abstract}

Keywords: reporte de caso , Miopatía, Homotoxicología, Medicina Biorreguladora, Homeopatía

\section{Introduction}

La miopatía inflamatoria (MI) es una afección poco frecuente que suele afectar al músculo estriado de forma aislada o más comúnmente, puede cursar con un compromiso de la piel y otros órganos, a menudo, los cuales, conducen a un deterioro severo de la calidad de vida. Tiene una incidencia anual de 0.8 - 8 casos/millón de habitantes y una prevalencia de $1-6$ pacientes/100.000 habitantes, siendo más frecuentes en mujeres con una relación 2:1, su grupo etario de presentación es en la infancia (10- 15 años) y edad adulta (45-60 años ) (Schmidt J,2018). Para el diagnóstico de una miopatia inflamatoria requiere de la exclusión de otras formas de miopatía que remedan una MI incluyendo las distrofias de cinturas, la distrofia facio-escápulo-humeral, así como miopatías metabólicas, mitocondriales, endocrinas e inducidas por drogas El hallazgo clínico más frecuente es la debilidad muscular simétrica de predominio proximal, que conlleva a dificultad para la marcha y para realizar cambios de movimientos como subir escaleras o levantar sillas u objetos. Las miopatías inflamatorias pueden dividirse en aquellas que tienen causa conocida o primarias y de causa indeterminada o idiopáticas. Sin embargo, actualmente de manera muy frecuente, la MI se presenta en ausencia de una causa identificable, haciendo plantear la existencia de una enfermedad primaria e idiopática del músculo. Estas miopatías inflamatorias idiopáticas (MII) son generalmente inmunomediadas, y suelen ocurrir en el contexto de una afección multisistémica, asociado a otras enfermedades autoinmunes, a la presencia de cáncer (paraneoplásico) o generadas por el uso de medicamentos miotóxicos.

\section{Timeline}




\section{0-10-13 - Paciente consulta al servicio de urgencias por cervicalgia leve asociado a disminucion de la fuerza en las cuatro extremidades, con importante limitación en la marcha, presentando aumento de la amplitud en su polígono de sustentación.}

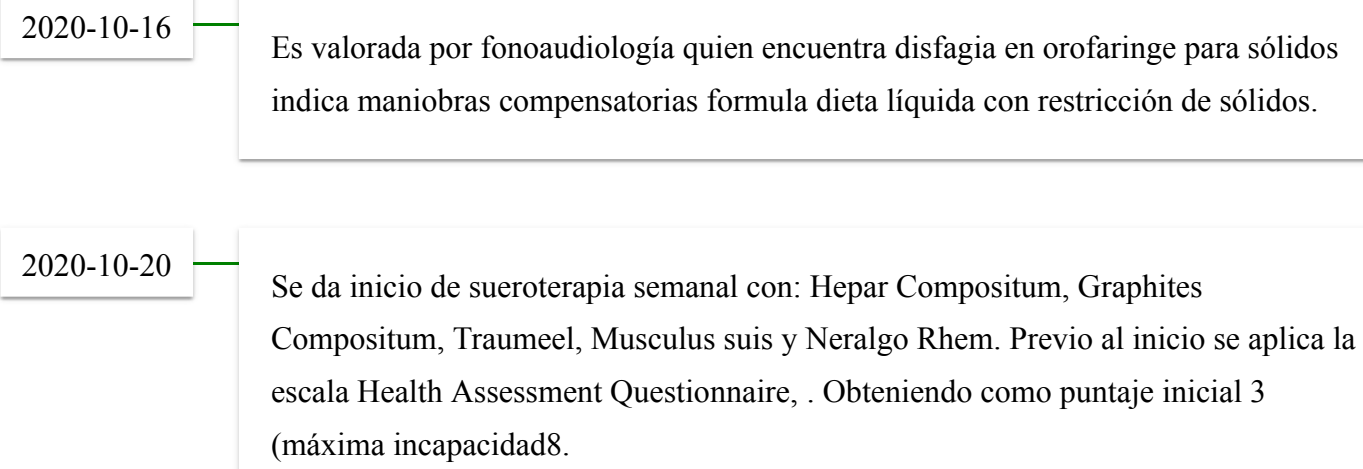

2020-10-29 - Se da egreso de hospitalizacion sin mejoria clinica . Por lo cual se decide inicio de detox oral (Lymphomyosot, Berbeel Hommaccord, Nuxeel Hommaccord) .

2020-12-04 - Se inicia reposicion de Vitamina D con histotal

2021-04-01 - Se decide inicio de Traumeel tópico para aplicar en áreas de la piel hiperpigmentadas.

2021-05-11 _ Hasta la fecha de presentación de este artículo, la paciente continúa en manejo con sueroterapia con: Traumeel, Cutis Compositum, Hepar Compositum, Musculus suis, Neralgo Rhem, Graphites Hommaccord.

\section{$\underline{\text { Narrative }}$}

Paciente femenino de 37 años de edad con antecedente de Síndrome de Steven Johnson en la adolescencia posterior a toma de isotretinoina durante 20 días por lesiones hiperpigmentadas en rostro. Consumo crónico de acetaminofén en horas de la noche para poder conciliar el sueño, sin otros antecedentes de importancia. Paciente quien consulta al servicio de urgencias en octubre del 2020 por cuadro clínico de aproximadamente 4 meses de evolución caracterizado por cervicalgía leve asociado a disminución de la fuerza muscular de origen central en los últimos 4 días previos a la consulta; el cuadro se asocia a limitación marcada y progresiva para la marcha. Al examen físico de ingreso se encuentra paciente con fuerza en miembros inferiores $2 / 5$, miembros superiores a nivel proximal $3 / 5$, a nivel distal $4 / 5$, hiporreflexia generalizada, respuesta plantar extensora 
bilateral, alteración en la deglución de sólidos, con importante limitación a la marcha con aumento del polígono de sustentación, limitación a los cambios de movimiento en decúbito supino, limitación al cambio de posición sentado - acostado y bipedestación.

\section{$\underline{\text { Diagnostics }}$}

\begin{tabular}{|c|c|c|c|}
\hline DATE & TYPE & VALUE & UNIT \\
\hline $2020-10-13$ & Creatine kinase & 2301 & $\mathrm{U} / \mathrm{L}$ \\
\hline 2020-10-13 & CRP (C-reactive protein) & 0.5 & $\mathrm{mg} / \mathrm{dL}$ \\
\hline 2020-10-13 & TSH (thyroid-stimulating hormone) & 6.38 & $\mathrm{mU} / \mathrm{L}$ \\
\hline 2020-10-14 & T4, free (thyroxine) & 1.38 & $\mathrm{nmol} / \mathrm{L}$ \\
\hline 2020-10-14 & Creatine kinase & 9220 & $\mathrm{U} / \mathrm{L}$ \\
\hline 2020-10-14 & Vitamin B12 & 1049 & $\mathrm{pg} / \mathrm{mL}$ \\
\hline $2020-10-15$ & AST (aspartate aminotransferase) & 311 & $\mathrm{IU} / \mathrm{L}$ \\
\hline $2020-10-15$ & LDH (lactate dehydrogenase) & 1291 & $\mathrm{IU} / \mathrm{L}$ \\
\hline $2020-10-15$ & LDH (lactate dehydrogenase) & 1291 & $\mathrm{IU} / \mathrm{L}$ \\
\hline $2020-10-15$ & BUN (blood urea nitrogen) & 4 & $\mathrm{mg} / \mathrm{dL}$ \\
\hline $2020-10-15$ & ALP (alkaline phosphatase) & 50 & $\mathrm{IU} / \mathrm{L}$ \\
\hline $2020-10-15$ & Creatinine & 0.5 & $\mathrm{mg} / \mathrm{dL}$ \\
\hline $2020-10-15$ & ALT (alanine aminotransferase) & 147 & $\mathrm{IU} / \mathrm{L}$ \\
\hline 2020-12-01 & Creatine kinase & 154 & $\mathrm{mg} / \mathrm{dL}$ \\
\hline 2020-12-01 & 25(OH)D (25-hydroxyvitamin D) & 15.6 & $\mathrm{ng} / \mathrm{mL}$ \\
\hline
\end{tabular}




\begin{tabular}{|c|c|c|c|}
\hline 2020-12-01 & Calcium & 7.4 & $\mathrm{mg} / \mathrm{dL}$ \\
\hline $2020-12-28$ & Creatinine & 0.24 & $\mathrm{mg} / \mathrm{dL}$ \\
\hline $2020-12-28$ & CRP (C-reactive protein) & 0.2 & $\mathrm{mg} / \mathrm{dL}$ \\
\hline $2020-12-28$ & ALT (alanine aminotransferase) & 38 & $\mathrm{IU} / \mathrm{L}$ \\
\hline $2020-12-28$ & Creatine kinase & 2672 & $\mathrm{U} / \mathrm{L}$ \\
\hline $2020-12-28$ & Uric acid & 4.9 & $\mathrm{mg} / \mathrm{dL}$ \\
\hline $2020-12-28$ & AST (aspartate aminotransferase) & 91 & $\mathrm{IU} / \mathrm{L}$ \\
\hline $2020-12-28$ & Creatinine & 0.24 & $\mathrm{mg} / \mathrm{dL}$ \\
\hline 2021-03-03 & Creatine kinase & 2683 & $\mathrm{U} / \mathrm{L}$ \\
\hline 2021-03-03 & CRP (C-reactive protein) & 0.13 & $\mathrm{mg} / \mathrm{dL}$ \\
\hline 2021-03-03 & ALT (alanine aminotransferase) & 26 & $\mathrm{IU} / \mathrm{L}$ \\
\hline 2021-03-03 & TSH (thyroid-stimulating hormone) & 10.29 & $\mathrm{mU} / \mathrm{L}$ \\
\hline 2021-03-03 & AST (aspartate aminotransferase) & 74 & $\mathrm{IU} / \mathrm{L}$ \\
\hline 2021-03-03 & Creatinine & 0.2 & $\mathrm{mg} / \mathrm{dL}$ \\
\hline 2021-05-11 & Creatine kinase & 1514 & $\mathrm{U} / \mathrm{L}$ \\
\hline 2021-05-11 & ALT (alanine aminotransferase) & 37 & $\mathrm{IU} / \mathrm{L}$ \\
\hline 2021-05-11 & TSH (thyroid-stimulating hormone) & 13 & $\mathrm{mU} / \mathrm{L}$ \\
\hline 2021-05-11 & Creatinine & 0.26 & $\mathrm{mg} / \mathrm{dL}$ \\
\hline
\end{tabular}




$\begin{array}{llll}2021-05-11 & \text { ALT (alanine aminotransferase) } & 37 & \mathrm{IU} / \mathrm{L} \\ 2021-05-11 & \text { CRP (C-reactive protein) } & 0.1 & \mathrm{mg} / \mathrm{dL} \\ 2021-10-13 & \text { Creatine kinase } & 7500 & \mathrm{U} / \mathrm{L} \\ 2021-10-13 & \text { BUN (blood urea nitrogen) } & 5 & \mathrm{mg} / \mathrm{dL} \\ 2021-10-13 & \text { Creatinine } & & \\ 2021-10-13 & \text { Glucose } & & 0.6 \\ \end{array}$

\section{Perspective}

Paciente en cuarta década de la vida, quien consulta por cuadro de disfagia de sólidos, debilidad muscular de cintura escapular y pelviana asociado a alteración de función hepática, CPK elevada, función renal normal, TSH elevada, para objetividad su función física se implementa la escala Health Assesment Questionnaure (HAQ) cuestionario con el cual se realiza una medición exhaustiva de la capacidad funcional de los pacientes con enfermedades reumatológicas. En este caso paciente obtiene puntaje inicial de 3 significando máxima incapacidad, intrahospitalariamente se inicia manejo con prednisolona $40 \mathrm{mg}$ la cual recibe solo por dos días y la paciente decide suspender por aparición de lesiones ulceradas en boca, por lo cual se ordena endoscopia de vías digestivas alta la cual reporta normal. Se inicia manejo con sueroterapia el día 20 de octubre al estar hospitalizada con posterior mejoría y aumento de fuerza muscular. Paciente por antecedente de mala tolerancia a medicamentos alopáticos, decide no recibir otro manejo convencional, acepta solo manejo biorregulador y fisioterapia, el 08 de enero fue valorada por Reumatología quien deja como diagnóstico polimiositis.

Actualmente paciente con mejoría progresiva de su patología, con recuperación total de independencia para realizar cambios de movimiento y marcha a pesar que continúa con aumento del polígono de sustentación, sin alteraciones para la deglución, con lesiones en piel con marcada sequedad en área de flexión, por lo cual se inicia manejo con traumeel tópico. Se aplica nuevamente escala HAQ obtiendo una una puntuacion de 1.38 , lo cual indica importante mejoria funcional en la paciente, en donde se entiende como puntuación 0 (sin incapacidad ) puntuación 3 (máxima incapacidad).

\section{Discussion}

Para iniciar se hablará de la etiopatogenia de la Miopatía Inflamatoria, ya que al tratarse de una enfermedad rara en su aparición, a lo largo de su historia se han sugerido que en su patogénica puede ser multifactorial tales como: fármacos, infecciones bacterianas, parasitarias o virales, hasta predisposiciones genéticas. En conjunto se ha encontrado que el gran punto de partida, es la 
alteración de la inmunidad tanto celular y humoral, la cual se ha ido documentando en los reportes de esas biopsias musculares en donde se encuentra un acumulo de linfocitos y anticuerpos específicos dirigidos contra moléculas citoplasmáticas implicadas en la síntesis de proteínas y la respuesta a agentes inmunosupresores. En la dermatomiositis se encuentra predominio de acumulo de linfocitos T CD4, linfocitos B y depósitos de las últimas fracciones del complemento. En la Polimiositis y en miopatía de cuerpos de inclusión se encuentran linfocitos T CD8 y macrófagos, invadiendo los miocitos. Esto parece indicar que la presencia de citocinas producto de este comportamiento inflamatorio son las responsables de la alteración en la función muscular manifestada clínica y para clínicamente. (Atluri R.,2016).

Es importante tener en cuenta que las dos principales presentaciones de miopatías inflamatorias son las polimiositis y dermatomiositis, en donde las manifestaciones cutáneas son el patrón diferenciador entre una y otra, en donde se puede cursar con lesiones patognomónicas como exantema heliotropo o pápulas de Gottron (Okiyama, N, 201.) Lesiones características como: lesiones descamativas o papulares en zonas de flexión o prominencia ósea y pueden cursar con fotosensibilidad la cual se manifiesta en el $50-80 \%$ de los pacientes. Dentro del compromiso muscular lo característico es la presencia de debilidad muscular simétrica de predominio proximal de las extremidades y de los músculos flexores anteriores del cuello, es su mayoría de manera simétrica asociados a síntomas como fatiga crónica y mialgias. Adicionalmente entre las manifestaciones se puede encontrar con disfagia proximal que refleja una afectación del músculo estriado de la faringe o del esófago proximal.

Teniendo en cuenta la fisiopatología y el comportamiento clínico de las miopatías inflamatorias se puede pensar que la paciente del caso en revisión, al cursar con síntomas de componente muscular y alteraciones en la piel estaría cursando con una dermatomiositis dado por la debilidad en la cintura pelviana: manifestada con la incapacidad para realizar actividades rutinarias, como levantarse de una silla sin apoyo y alteraciones en la marcha. La afección de la cintura escapular se manifiesta con incapacidad para elevar los brazos por encima de la cabeza, disfagia de sólidos, manifestaciones cutáneas dadas por lesiones descamativas en región flexora, las cuales, en este caso, no son las típicas lesiones cutáneas, por presencia de lesiones secuelares del Síndrome de Steven Johnson padecido por la paciente hace 16 años. Dentro de este contexto es importante saber que como hallazgos de laboratorio se pueden encontrar niveles de enzimas musculares alterados, siendo la elevación de la creatin fosfokinasa (CPK) el indicador más sensible y específico de la enfermedad muscular activa. La aldolasa sérica es un indicador menos sensible para detectar la miositis activa, sin embargo, sus niveles pueden estar elevados en presencia de CPK normal. Las transaminasas hepáticas (GOT, GPT) y la lactato deshidrogenasa (LDH) pueden ser elevadas, pero son poco específicas y están relacionadas con la presencia de disfunción muscular. (Mammen A. L, 2016) . Dentro de los hallazgos en la electromiografía se evidencia el aumento de la irritabilidad de la membrana de las células musculares estriadas. Adicionalmente es importante tener en cuenta que la biopsia muscular tanto en la polimiositis como dermatomiositis muestra necrosis del tejido muscular, asociado a degeneración, regeneración y cambios inflamatorios de las fibras musculares. (Acosta, I,2019).

Es importante tener en cuenta que el tratamiento estudiado para las miopatías inflamatorias como marco principal está basado en corticoesteroides sistémicos, inmunoglobulinas intravenosas o 
fármacos inmunosupresores como metotrexato o azatioprina. Es aquí donde la paciente al ver poca tolerancia a la prednisolona se decide iniciar manejo con Medicina Biorreguladora de sistemas, la cual está orientada a dar manejo de las redes biológicas desreguladas con medicamentos homeopáticos, los cuales buscan mejorar la calidad de vida del paciente. Se encuentra un paciente con importante marcador inflamatorio que genera ruptura o disfunción en la cadena de transmisión de información celular, matriz extracelular y SNC. Por ellos es importante tener claro el papel de la matriz extracelular que es entendida como el medio en el que se almacena y degradan diferentes toxinas, siendo punto de partida conjunto con alteraciones mitocondriales para generar proceso de disfunción y disregulación celular. Y la inflamación es un elemento común denominador ante cualquier trastorno funcional o estructural.

Con lo anterior es importante entender que el organismo tiene tres niveles de respuesta a tales discusiones conocidas como: 1. Respuesta inmediata de tipo humoral, la cual busca favorecer los proceso fisiológicos de eliminación (sudoración o secreciones), 2. Afectación de la matriz extracelular cuya rigidez y acumulo excesivo de toxinas provocaría disfunción del tejido afectado, en donde ya entraría a una evolución crónica y procesos degenerativos por disfunción de los sistemas de autorregulación, 3. Una respuesta celular que puede cursar con una fase de degeneración, la cual implica un daño severo a las estructuras intracelulares y limitación en la eliminación de toxinas y una fase de desdiferenciación.

Es así cómo se decide iniciar manejo con terapia biorreguladora con detox básico oral en busca de mejorar el terreno y favorecer la eliminación de toxinas de la matriz, y se asocia a sueroterapia con:

Traumeel para manejo de la inflamación crónica.

Hepar Compositum. para manejo de hepatopatía crónica evidenciada por la alteración en la función hepática y bajo tolerancia al uso de medicamentos alopáticos.

Cutis Compositum. por lesiones cutáneas asociadas como secuelas del Síndrome de Steven Johnson.

Musculus suis para favorecer la desinflamación y recuperación del tejido muscular afectado.

Neralgo Rhem para manejo de polineuropatía severa documentada en electromiografía.

Graphites Homm. por presencia de fibrosis y rigidez de la piel, la cual muy seguramente se puede extender a tejido celular subcutáneo y alcanza planos musculares

\section{Conclusion}

Con este caso de miopatia inflamatoria podemos encontrar que el manejo con medicina biorreguladora demostró un gran impacto en su calidad de vida, al poder recuperar mas del $50 \%$ de su capacidad funcional, lo cual a significado en restablecimiento en su independencia en la marcha y realización de sus actividades diarias y lo más importante, sin presentar efectos secundarios o complicaciones por uso de terapia bioreguladora, situación que no se logró obtener con medicina tradicional. En este caso clínico se puede encontrar los factores etiopatogénicos que favorecieron y 
facilitaron la alteración de su matriz conllevando a un proceso inflamatorio crónico de bajo grado que finalmente se manifiesta con la degeneración y necrosis de fibras musculares y es donde la medicina biorreguladora en su proceso hormético logra dar inicio de. la reparación y recuperación funcional en $80 \%$ de todas las habilidades motrices de la paciente, por lo cual se puede concluir que la medicina biorreguladora puede dar resultados prometedores en manejo de estas patologías idiopáticas .

\section{$\underline{\text { Acknowledgements }}$}

Agradecemos en primera instancia a nuestra paciente por habernos dado la oportunidad de dar a conocer a travez de su caso, el impacto tan positivo que tiene la medicina bioreguladora en este tipo de patologías.

También damos gracias a nuestros maestros que nos brindaron las heramientas necesarias para poder definir cual era el manejo mas indicado en nuestra paciente para poder impactar positivamente en su vida .

\section{$\underline{\text { References }}$}

1- Schmidt J. (2018). Current Classification and Management of Inflammatory Myopathies. Journal of neuromuscular diseases, 5(2), 109-129.

2. Acosta, I., Matamala, J. M., Jara, P., Pino, F., Gallardo, A., \& Verdugo, R. (2019). Miopatías inflamatorias idiopáticas: una mirada actualizada al diagnóstico y el manejo [Idiopathic inflammatory myopathies. A review]. Revista médica de Chile, 147(3), 342-355.

3. Atluri R. B. (2016). Inflammatory Myopathies. Missouri medicine, 113(2), 127-130.

4. Okiyama, N. , \& Fujimoto, M. (2019). Cutaneous manifestations of dermatomyositis characterized by myositisspecific autoantibodies. F1000Research, 8, F1000 Faculty Rev-1951.

5. Mammen A. L. (2016). Autoimmune Myopathies. Continuum (Minneapolis, Minn.), 22(6, Muscle and Neuromuscular Junction Disorders), 1852-1870. https://doi.org/10.1212/01.CON.0000511070.50715.ab

6. Schneider C. (2011). Traumeel - an emerging option to nonsteroidal anti-inflammatory drugs in the management of acute musculoskeletal injuries. International journal of general medicine.

\section{Attachments}




\begin{tabular}{|c|c|}
\hline \multirow{2}{*}{$\begin{array}{l}13 \text { de Octubre } \\
2020\end{array}$} & $\begin{array}{l}\text { TAC de cráneo simple: normal y TAC de columna cervical: Normal. Radiografía } \\
\text { de Tórax: Normal }\end{array}$ \\
\hline & $\begin{array}{l}\text { VSG: } 3 \text { VDRL: No reactiva TSH: } 6.38 \text { ANAs: Positivo } 1 / 160 \text { VIH: Negativo INR: } \\
\text { 1.1 TPT: } 25 \text { Hemograma: Leucos: } 8.35 \text { Neutrófilos: } 68.4 \% \text { Hb: } 15.3 \text { VCM: } 83 \\
\text { Plaquetas: } 417\end{array}$ \\
\hline $\begin{array}{l}14 \text { de Octubre } \\
2020\end{array}$ & $\begin{array}{l}\text { CPK } 9220 \text { Vitamina B12: } 1049 \text { Tiroxina T4 libre: } 1.38 \text { Anti DD ro y La: Negativo } \\
\text { C3: } 120 \text { negativo C4: } 20,5 \text { Negativo }\end{array}$ \\
\hline $\begin{array}{l}15 \text { de octubre } \\
2020\end{array}$ & $\begin{array}{l}\text { RNM cráneo contrastado: Normal RNM Columna Cervical: Normal Aldolasa: } \\
84.1\end{array}$ \\
\hline $\begin{array}{l}16 \text { de octubre } \\
2020\end{array}$ & $\begin{array}{l}\text { Valoración por fonoaudiología: Disfagia orofaringe para sólidos indica } \\
\text { maniobras compensatorias, indican dieta líquida restricción de sólidos. }\end{array}$ \\
\hline $\begin{array}{l}19 \text { de noviembre } \\
2020\end{array}$ & $\begin{array}{l}\text { Se recibe reporte de biopsia de músculo: Miopatía necrotizante compatible con } \\
\text { miopatía inflamatoria y atrofia selectiva de fibras tipo II. }\end{array}$ \\
\hline $\begin{array}{l}30 \text { de noviembre } \\
2020\end{array}$ & $\begin{array}{l}\text { Electromiografía de } 4 \text { extremidades: compromiso axonal de los nervios } \\
\text { mediano, cubital, peroneo y tibial bilateral, compatible con polineuropatía } \\
\text { severa. }\end{array}$ \\
\hline $\begin{array}{l}01 \text { Diciembre } \\
2020\end{array}$ & GOT: 10.5 GPT: 11.5 Fósforo: 1.67 \\
\hline $\begin{array}{l}28 \text { Diciembre } \\
2020\end{array}$ & $\begin{array}{l}\text { Hemograma: Hb: } 16 \text { Leucocitos: } 6.4 \text { Neutrófilos } 36 \% \text { Plaquetas: } 421 \text { ANAs: } \\
\text { Positivo 1/160, patrón: moteado, Ac SS1 Ro: } 5.5 \text { (Negativo) Ac Sm:1.9 } \\
\text { (Negativo) Varicela Zoster lgG: } 36.0 \text { CPK: } 2672 \text { Factor Reumatoideo: } 7 \\
\text { Antígeno de superficie Hepatitis B: } 0.24 \text { Anticuerpo Hepatitis C: } 0.073 \\
\text { Uroanálisis: cristales abundantes de oxalato de calcio }\end{array}$ \\
\hline $\begin{array}{l}29 \text { Diciembre } \\
2020\end{array}$ & Aldolasa: 28.1 ANCAs: Negativo AMA: 36.5 (Positivo) \\
\hline 03 de Marzo 2021 & Hemograma: Hb: 15.7Hto: 49,2 Leucos: 9.06 Neutrófilos: $50.7 \%$ \\
\hline 11 de Mayo 2021 & Aldolasa: 18.7 \\
\hline
\end{tabular}




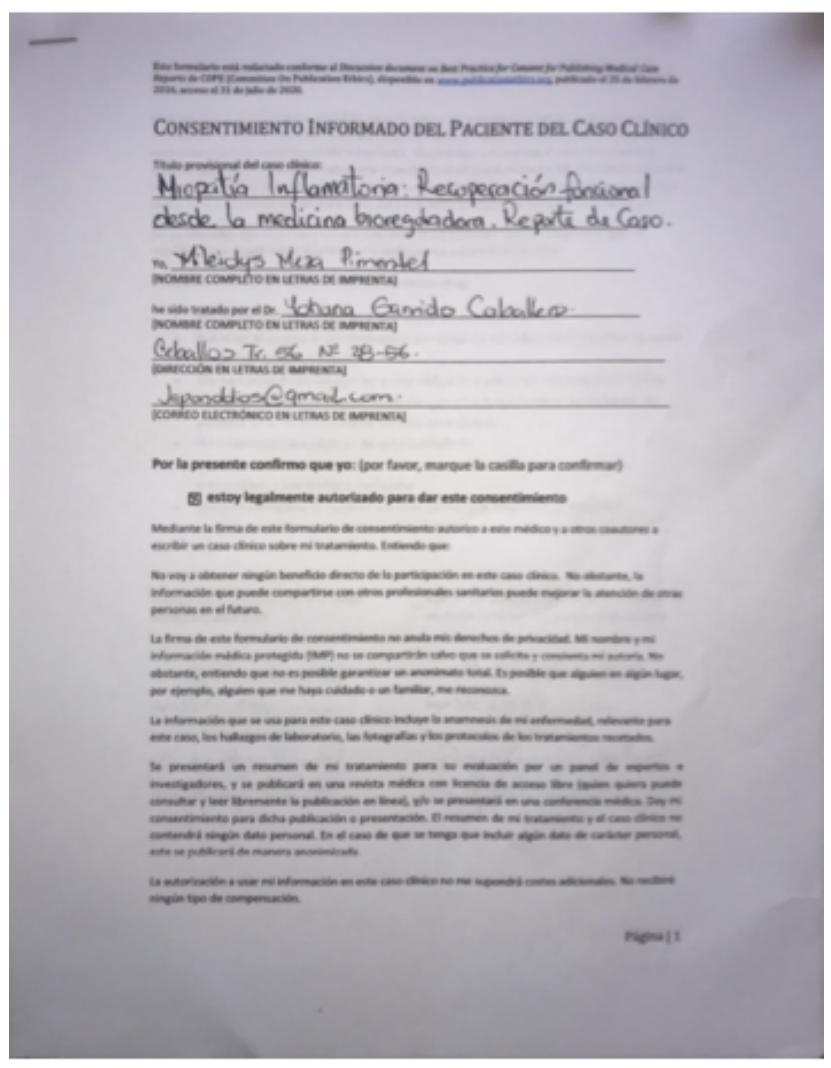

Escaneado con CamScanner

Consentimiento Informado Paciente 